\title{
Estimation Based on Generalized Order Statistics from a Mixture of Two Rayleigh Distributions
}

\author{
Tahani A. Abushal ${ }^{1} \&$ Areej M. AL-Zaydi ${ }^{2}$ \\ ${ }^{1}$ Math. Dept., Umm AL-Qura University, Makkah, Saudi Arabia \\ ${ }^{2}$ Math. Dept., Taif University, Taif, Saudi Arabia \\ Correspondence: Tahani A. Abushal, Math. Dept., Umm AL-Qura University, PO box 9324, Makkah 21955, Saudi \\ Arabia. Tel: 966-2558-0200. E-mail: Taabushal@uqu.edu.sa
}

Received: March 30, 2012 Accepted: April 11, 2012 Online Published: July 30, 2012

doi:10.5539/ijsp.v1n2p79 URL: http://dx.doi.org/10.5539/ijsp.v1n2p79

\begin{abstract}
This article is concerned with the problem of estimating the parameters, reliability and hazard rate functions of the mixture of two Rayleigh distributions (MTRD) based on generalized order statistics (GOS). The maximum likelihood and Bayes methods of estimation are used for this purpose. The Markov chain Monte Carlo (MCMC) method is used for obtaining the Bayes estimates under the squared error loss and LINEX loss functions. Our results are specialized to progressive Type-II censored order statistics and upper record values. Comparisons are made between Bayesian and maximum likelihood estimators via a Monte Carlo simulation study.
\end{abstract}

Keywords: generalized order statistics, Bayes estimation, progressive censoring, Markov chain Monte Carlo, Monte Carlo simulation

\section{Introduction}

The Rayleigh distribution $(R D)$ was first derived by Lord Rayleigh in connection with a study of acoustical problems. Since then many investigators have used the $R D$ or some related forms of it in a variety of engineering, wave propagation, radiation and analysis of target data studies. The $R D$ is also used to model wave heights in oceanography, and in communication theory to describe hourly median and instantaneous peak power of received radio signals. Several such situations have been discussed by Polovko (1968), Takeshi Yamane (1998), Zhi Ren et al. (2011), and many others. The $R D$ is a special case of two parameter Weibull distribution. A random variable $T$ is said to have a $R D$ with parameter $\theta$ if its probability density function $(P D F)$ is given by

$$
f(t)=2 \theta t e^{-\theta t^{2}}, t>0,(\theta>0) .
$$

The cumulative distribution function $(C D F)$, reliability function $(R F)$ and the hazard rate function $(H R F)$ are given, respectively, by

$$
\begin{gathered}
F(t)=1-e^{-\theta t^{2}}, t>0,(\theta>0), \\
R(t)=e^{-\theta t^{2}}, \\
H(t)=2 \theta t,
\end{gathered}
$$

where $H(\cdot)=\frac{f(\cdot)}{R(\cdot)}$.

Mixtures of distributions arise frequently in life testing, reliability, biological and physical sciences. Some of the most important references that discussed different types of mixtures of distributions are a monograph by Everitt and Hand (1981), Titterington et al. (1985) and McLachlan and Basford (1988). Bayesian inferences based on finite mixture distribution have been discussed by several authors. Papadapoulos and Padgett (1986) considered Bayesian estimation of the mixing parameter, mean and reliability function of a mixture of two exponential lifetime distributions based on right censored samples. Attia (1993) considered the MTRD and obtained estimates of model parameters using maximum likelihood $(M L)$ and Bayesian approach with censored sampling. Ahmad et al. (1997) derive approximate Bayes estimation for mixture of two Weibull distributions under Type-II censoring. Jaheen (2005b) considered estimation for the mixed exponential distribution based on record statistics. Soliman (2006) obtained the estimates of the parameters and functions of these parameters of the MTRD based on progressively 
Type-II censored samples when the mixing proportion $p$ is known. Saleem and Aslam (2008a \& b) use ordinary type I right censored data for Bayesian analysis of Rayleigh mixture. Saleem and Irfan (2010) studied some properties of the Bayes estimates of the Rayleigh mixture parameters. The PDF, CDF, RF and $H R F$ of the $M T R D$ are given, respectively, by

$$
\begin{gathered}
f(t)=p_{1} f_{1}(t)+p_{2} f_{2}(t), \\
F(t)=p_{1} F_{1}(t)+p_{2} F_{2}(t), \\
R(t)=p_{1} R_{1}(t)+p_{2} R_{2}(t), \\
H(t)=\frac{f(t)}{R(t)},
\end{gathered}
$$

where, for $j=1,2$, the mixing proportions $p_{j}$ are such that $0 \leq p_{j} \leq 1, p_{1}+p_{2}=1$ and $f_{j}(t), F_{j}(t), R_{j}(t)$ are given from (1), (2), (3) after using $\theta_{j}$ instead of $\theta$.

The property of identifiability is an important consideration on estimating the parameters in a mixture of distributions. Also, testing hypothesis, classification of random variables, can be meaning fully discussed only if the class of all finite mixtures is identifiable. Identifiability of mixtures has been discussed by several authors, including Teicher (1963), AL-Hussaini and Ahmad (1981) and Ahmad (1988).

The concept of GOS was introduced by Kamps (1995) to unify several important ordering concepts that were separately treated in statistical literature, such as ordinary order statistics, ordinary record values, progressive Type-II censored order statistics and sequential order statistics, among others. For various distributional properties of $G O S$, see Kamps (1995). The GOS have been considered extensively by many authors, among others, they are Ahsanullah (1996; 2000), Kamps and Gather (1997), Cramer and Kamps (2000), Habibullah and Ahsanullah (2000), Jaheen (2002; 2005a), AL-Hussaini and Ahmad (2003), AL-Hussaini (2004), Ahmad (2007; 2008), Aboeleneen (2010), Jaheen and Al Harbi (2010), Abu El Fotouh (2011) and Ateya and Ahmad (2011).

The purpose of this paper is to estimate the parameters, $R F$ and $H R F$ of the MTRD based on GOS using ML and Bayes methods. The non-informative prior and the conjugate prior are assumed to carry out the Bayesian analysis. The results are specialized to progressive Type-II censored order statistics and record values. This paper is organized as follows: In Section 2, the $M L$ estimators of parameters, $R F$ and $H R F$ of the $M T R D$ are derived. In Section 3, the $M C M C$ method is used for obtaining the Bayes estimators of parameters, $R F$ and $H R F$ of the $M T R D$ under the squared error loss and LINEX loss functions. Comparisons between Bayesian and $M L$ estimators via Monte Carlo simulation study are made in Section 4. Finally, Concluding remarks about comparisons between the estimators are considered in Section 5.

\section{Maximum Likelihood Estimation}

Let $T_{1 ; n, \widetilde{m}, k}, T_{2 ; n, \widetilde{m}, k}, \ldots, T_{n ; n, \widetilde{m}, k}, k>0, \widetilde{m}=\left(m_{1}, \ldots, m_{n-1}\right) \in \mathfrak{R}^{n-1}, m_{1}, \ldots, m_{n-1} \in \mathfrak{R}$, are $n G O S$ drawn from the $M T R D$. The likelihood function ( $L F)$ is given in (Kamps, 1995), for $-\infty<t_{1}<\ldots<t_{n}<\infty$, by

$$
L(\theta \mid \mathbf{t})=k \prod_{i=1}^{n-1} \gamma_{i}\left\{\prod_{i=1}^{n-1}\left[R\left(t_{i}\right)\right]^{m_{i}} f\left(t_{i}\right)\right\}\left[R\left(t_{n}\right)\right]^{k-1} f\left(t_{n}\right)
$$

where $\mathbf{t}=\left(t_{1}, \ldots, t_{n}\right), \theta \in \Theta, \Theta$ is the parameter space, and

$$
\gamma_{i}=k+n-i+M_{i}>0, M_{i}=\sum_{v=i}^{n-1} m_{v} .
$$

Substituting (1), (1) in (5), the $L F$ takes the form

$$
L(\theta \mid \mathbf{t}) \propto \prod_{i=1}^{n-1}\left[p_{1} R_{1}\left(t_{i}\right)+p_{2} R_{2}\left(t_{i}\right)\right]^{m_{i}} \prod_{i=1}^{n}\left[p_{1} f_{1}\left(t_{i}\right)+p_{2} f_{2}\left(t_{i}\right)\right]\left[p_{1} R_{1}\left(t_{n}\right)+p_{2} R_{2}\left(t_{n}\right)\right]^{k-1} .
$$

Take the logarithm of (6), we have

$$
\ell(\theta) \equiv \ln L(\theta \mid \mathbf{t}) \propto \sum_{i=1}^{n-1} m_{i} \ln \left[p_{1} R_{1}\left(t_{i}\right)+p_{2} R_{2}\left(t_{i}\right)\right]+\sum_{i=1}^{n} \ln \left[p_{1} f_{1}\left(t_{i}\right)+p_{2} f_{2}\left(t_{i}\right)\right]
$$




$$
+(k-1) \ln \left[p_{1} R_{1}\left(t_{n}\right)+p_{2} R_{2}\left(t_{n}\right)\right],
$$

where $p_{1}=p, p_{2}=1-p$.

Differentiating (7) with respect to the parameters $p$ and $\theta_{j}$ and equating to zero gives the following likelihood equations

$$
\left.\begin{array}{l}
\frac{\partial \ell}{\partial p}=\sum_{i=1}^{n-1} m_{i} \vartheta^{*}\left(t_{i}\right)+\sum_{i=1}^{n} \vartheta\left(t_{i}\right)+(k-1) \vartheta^{*}\left(t_{n}\right)=0, \\
\frac{\partial \ell}{\partial \theta_{j}}=p_{j}\left\{\sum_{i=1}^{n} \xi_{j}\left(t_{i}\right) \psi_{j}\left(t_{i}\right)-\sum_{i=1}^{n-1} m_{i} \psi_{j}^{*}\left(t_{i}\right)-(k-1) \psi_{j}^{*}\left(t_{n}\right)\right\}=0, \quad j=1,2
\end{array}\right\},
$$

where, for $j=1,2$

$$
\left.\begin{array}{ll}
\vartheta\left(t_{i}\right)=\frac{f_{1}\left(t_{i}\right)-f_{2}\left(t_{i}\right)}{f\left(t_{i}\right)}, & \vartheta^{*}\left(t_{i}\right)=\frac{R_{1}\left(t_{i}\right)-R_{2}\left(t_{i}\right)}{R\left(t_{i}\right)}, \\
\psi_{j}\left(t_{i}\right)=\frac{f_{j}\left(t_{i}\right)}{f\left(t_{i}\right)}, & \psi_{j}^{*}\left(t_{i}\right)=\frac{t_{i}^{2} R_{j}\left(t_{i}\right)}{R\left(t_{i}\right)}, \\
\xi_{j}\left(t_{i}\right)=\left[\frac{1}{\theta_{j}}-t_{i}^{2}\right] &
\end{array}\right\} .
$$

Equations (8) do not yield explicit solutions for $p$ and $\theta_{j}, j=1,2$, and have to be solved numerically to obtain the $M L$ estimates of the three parameters, Newton-Raphson iteration is employed to solve (8). The corresponding $M L$ estimates of the reliability function $R(t)$ and the Hazard rate function $H(t)$ are given respectively by (3) and (4) after replacing $p, \theta_{1}$ and $\theta_{2}$ by their $M L$ estimates $\hat{p}, \hat{\theta}_{1}$ and $\hat{\theta}_{2}$, (the solution of the above nonlinear equations).

\section{Prior, Posterior and Bayes Estimators}

Recently, there has been a considerable amount of interest in the Bayesian approach in estimation and reliability studies. It has received frequent attention for analyzing failure data and other time-to-event data, and has been often proposed as a valid alternative to traditional statistical perspectives. The Bayesian approach to estimation of the parameters and reliability analysis allows prior subjective knowledge on lifetime parameters and technical information on the failure mechanism, as well as experimental data, to be incorporated into the inferential procedure. Bayesian methods usually require less sample data to achieve the same quality of inferences than methods based on sampling theory. In this section, we present the Bayesian estimation for the parameters, $R F$ and $H R F$ for $M T R D$ based on GOS. In this section, Bayesian estimation for the parameters of the MTRD is considered under squared error and LINEX (Linear-Exponential) loss functions.

\subsection{Bayes Estimation Using Conjugate Prior}

Let $p, \theta_{1}$ and $\theta_{2}$ are independent random variables such that $p \sim \operatorname{Beta}\left(b_{1}, b_{2}\right)$ and for $j=1,2, \theta_{j}$ to follow an inverted gamma prior distribution with $P D F$

$$
\pi\left(\theta_{j}\right)=\frac{1}{\Gamma\left(\alpha_{j}\right)}\left(\frac{\alpha_{j}}{\beta_{j}}\right)^{\alpha_{j}} \theta_{j}^{\alpha_{j}-1} \exp \left[-\frac{\alpha_{j}}{\beta_{j}} \theta_{j}\right], \quad\left(\theta_{j}, \alpha_{j}, \beta_{j}>0\right) .
$$

A joint prior density function of $\theta=\left(p, \theta_{1}, \theta_{2}\right)$ is then given by

$$
\begin{gathered}
\pi(\theta)=\pi_{1}(p) \pi_{2}\left(\theta_{1}\right) \pi_{3}\left(\theta_{2}\right), \\
\pi(\theta) \propto p_{1}^{b_{1}-1} p_{2}^{b_{2}-1} \prod_{j=1}^{2} \theta_{j}^{\alpha_{j}-1} \exp \left[-\Sigma_{j=1}^{2} \frac{\alpha_{j}}{\beta_{j}} \theta_{j}\right],
\end{gathered}
$$

where $0<p_{1}<1, p_{2}=1-p_{1}$ and for $j=1,2 \theta_{j}>0,\left(b_{j}, \alpha_{j}, \beta_{j}\right)>0$.

It follows, from (6) and (11), that the joint posterior density function is given by

$$
\begin{aligned}
\pi^{*}(\theta \mid \mathbf{t})=A_{1} & p_{1}^{b_{1}-1} p_{2}^{b_{2}-1} \prod_{j=1}^{2} \theta_{j}^{\alpha_{j}-1} \exp \left[-\Sigma_{j=1}^{2} \frac{\alpha_{j}}{\beta_{j}} \theta_{j}\right] \prod_{i=1}^{n-1}\left[p_{1} R_{1}\left(t_{i}\right)+p_{2} R_{2}\left(t_{i}\right)\right]^{m_{i}} \\
& \times \prod_{i=1}^{n}\left[p_{1} f_{1}\left(t_{i}\right)+p_{2} f_{2}\left(t_{i}\right)\right]\left[p_{1} R_{1}\left(t_{n}\right)+p_{2} R_{2}\left(t_{n}\right)\right]^{k-1},
\end{aligned}
$$


where

$$
A_{1}^{-1}=\int_{\theta} \pi(\theta) L(\theta \mid \mathbf{t}) d \theta
$$

Under the squared error loss and LINEX loss functions, the Bayes estimator of a function, say $\phi \equiv \phi\left(p, \theta_{1}, \theta_{2}\right)$, are given, respectively, by

$$
\begin{gathered}
\hat{\phi}_{B S}=E(\phi \mid \mathbf{t})=\int_{\theta} \phi \quad \pi^{*}(\theta \mid \mathbf{t}) d \theta, \\
\hat{\phi}_{B L}=-\frac{1}{a} \ln \left[E\left(e^{-a \phi} \mid \mathbf{t}\right)\right]=-\frac{1}{a} \ln \left[\int_{\theta} e^{-a \phi} \quad \pi^{*}(\theta \mid \mathbf{t}) d \theta\right],
\end{gathered}
$$

where the integral is taken over the three dimensional space and $a \neq 0$. To compute the integral we propose to consider MCMC methods.

The conditional posterior distribution of the parameters $p, \theta_{1}$ and $\theta_{2}$ using conjugate prior can be computed and written, respectively, by

$$
\begin{aligned}
& \pi^{*}\left(p \mid \theta_{1}, \theta_{2}, \mathbf{t}\right) \propto p_{1}^{b_{1}-1} p_{2}^{b_{2}-1} \prod_{i=1}^{n-1}\left[p_{1} R_{1}\left(t_{i}\right)+p_{2} R_{2}\left(t_{i}\right)\right]^{m_{i}} \prod_{i=1}^{n}\left[p_{1} f_{1}\left(t_{i}\right)+p_{2} f_{2}\left(t_{i}\right)\right] \times\left[p_{1} R_{1}\left(t_{n}\right)+p_{2} R_{2}\left(t_{n}\right)\right]^{k-1}, \\
& \pi^{*}\left(\theta_{1} \mid p, \theta_{2}, \mathbf{t}\right) \propto \theta_{1}^{\alpha_{1}-1} e^{-\alpha_{1} \theta_{1} / \beta_{1}} \prod_{i=1}^{n-1}\left[p_{1} R_{1}\left(t_{i}\right)+p_{2} R_{2}\left(t_{i}\right)\right]^{m_{i}} \prod_{i=1}^{n}\left[p_{1} f_{1}\left(t_{i}\right)+p_{2} f_{2}\left(t_{i}\right)\right] \times\left[p_{1} R_{1}\left(t_{n}\right)+p_{2} R_{2}\left(t_{n}\right)\right]^{k-1}, \\
& \pi^{*}\left(\theta_{2} \mid p, \theta_{1}, \mathbf{t}\right) \propto \theta_{2}^{\alpha_{2}-1} e^{-\alpha_{2} \theta_{2} / \beta_{2}} \prod_{i=1}^{n-1}\left[p_{1} R_{1}\left(t_{i}\right)+p_{2} R_{2}\left(t_{i}\right)\right]^{m_{i}} \prod_{i=1}^{n}\left[p_{1} f_{1}\left(t_{i}\right)+p_{2} f_{2}\left(t_{i}\right)\right] \times\left[p_{1} R_{1}\left(t_{n}\right)+p_{2} R_{2}\left(t_{n}\right)\right]^{k-1} .
\end{aligned}
$$

\subsection{Bayes Estimation Using Non-informative Prior}

Assuming that all of the parameters consisting $\theta$ are positive and independent, and that we are indifferent about the prior information about $\theta$ so that we set improper non-informative prior to $\theta_{j}, j=1,2$, and $p$ as follows

$$
\pi_{1}(p) \propto \frac{1}{p}, \quad \pi_{2}\left(\theta_{1}\right) \propto \frac{1}{\theta_{1}}, \quad \pi_{3}\left(\theta_{2}\right) \propto \frac{1}{\theta_{2}} .
$$

so that

$$
\begin{gathered}
\pi(\theta) \propto \pi_{1}(p) \pi_{2}\left(\theta_{1}\right) \pi_{3}\left(\theta_{2}\right), \\
\pi(\theta) \propto\left(p \theta_{1} \theta_{2}\right)^{-1}, \quad\left(0<p<1, \theta_{1}, \theta_{2}>0\right) .
\end{gathered}
$$

The posterior density function can be obtained from (6) and (19), as

$$
\pi^{*}(\theta \mid \mathbf{t})=A_{1}\left(p \theta_{1} \theta_{2}\right)^{-1} \prod_{i=1}^{n-1}\left[p_{1} R_{1}\left(t_{i}\right)+p_{2} R_{2}\left(t_{i}\right)\right]^{m_{i}} \times \prod_{i=1}^{n}\left[p_{1} f_{1}\left(t_{i}\right)+p_{2} f_{2}\left(t_{i}\right)\right]\left[p_{1} R_{1}\left(t_{n}\right)+p_{2} R_{2}\left(t_{n}\right)\right]^{k-1} .
$$

Under the squared error loss and LINEX loss functions, the Bayes estimator of a function, say $\phi \equiv \phi\left(p, \theta_{1}, \theta_{2}\right)$, are given, respectively, by

$$
\begin{gathered}
\hat{\phi}_{B S}=E(\phi \mid \mathbf{t})=\int_{\theta} \phi \quad \pi^{*}(\theta \mid \mathbf{t}) d \theta, \\
\hat{\phi}_{B L}=-\frac{1}{a} \ln \left[E\left(e^{-a \phi} \mid \mathbf{t}\right)\right]=-\frac{1}{a} \ln \left[\int_{\theta} e^{-a \phi} \quad \pi^{*}(\theta \mid \mathbf{t}) d \theta\right],
\end{gathered}
$$

where the integral is taken over the three dimensional space and $a \neq 0$. To compute the integral we propose to consider MCMC methods.

The conditional posterior distribution of the parameters $p, \theta_{1}$ and $\theta_{2}$ using non-informative prior can be computed and written, respectively, by

$$
\pi^{*}\left(p \mid \theta_{1}, \theta_{2}, \mathbf{t}\right) \propto p^{-1} \prod_{i=1}^{n-1}\left[p_{1} R_{1}\left(t_{i}\right)+p_{2} R_{2}\left(t_{i}\right)\right]^{m_{i}} \prod_{i=1}^{n}\left[p_{1} f_{1}\left(t_{i}\right)+p_{2} f_{2}\left(t_{i}\right)\right] \times\left[p_{1} R_{1}\left(t_{n}\right)+p_{2} R_{2}\left(t_{n}\right)\right]^{k-1},
$$




$$
\begin{aligned}
& \pi^{*}\left(\theta_{1} \mid p, \theta_{2}, \mathbf{t}\right) \propto \theta_{1}^{-1} \prod_{i=1}^{n-1}\left[p_{1} R_{1}\left(t_{i}\right)+p_{2} R_{2}\left(t_{i}\right)\right]^{m_{i}} \prod_{i=1}^{n}\left[p_{1} f_{1}\left(t_{i}\right)+p_{2} f_{2}\left(t_{i}\right)\right] \times\left[p_{1} R_{1}\left(t_{n}\right)+p_{2} R_{2}\left(t_{n}\right)\right]^{k-1}, \\
& \pi^{*}\left(\theta_{2} \mid p, \theta_{1}, \mathbf{t}\right) \propto \theta_{2}^{-1} \prod_{i=1}^{n-1}\left[p_{1} R_{1}\left(t_{i}\right)+p_{2} R_{2}\left(t_{i}\right)\right]^{m_{i}} \prod_{i=1}^{n}\left[p_{1} f_{1}\left(t_{i}\right)+p_{2} f_{2}\left(t_{i}\right)\right] \times\left[p_{1} R_{1}\left(t_{n}\right)+p_{2} R_{2}\left(t_{n}\right)\right]^{k-1} .
\end{aligned}
$$

\subsection{MCMC Method}

In this subsection, the $M C M C$ method is considered to compute the Bayes estimators of the parameters $p, \theta_{1}$ and $\theta_{2}$. We consider the $M C M C$ techniques, namely the Metropolis-Hasting algorithm, to generate samples from the conditional posterior distributions and then compute the Bayes estimates. For more details about the $M C M C$ methods see, for example, Upadhyay et al. (2001), Press (2003) and Upadhyay and Gupta (2010). The MetropolisHastings algorithm generate samples from an arbitrary proposal distribution (i.e. a Markov transition kernel). The following MCMC procedure is proposed to compute Bayes estimators of $\phi \equiv \phi\left(p, \theta_{1}, \theta_{2}\right)$ based on squared error and LINEX loss functions.

1) Start with initial guess of $p, \theta_{1}$ and $\theta_{2}$ say $p^{0}, \theta_{1}^{0}$ and $\theta_{2}^{0}$, respectively.

2) Set $i=1$.

3) Generate $p$ from $\pi^{*}\left(p \mid \theta_{1}, \theta_{2}, \mathbf{t}\right), \theta_{1}$ from $\pi^{*}\left(\theta_{1} \mid p, \theta_{2}, \mathbf{t}\right)$. and $\theta_{2}$ from $\pi^{*}\left(\theta_{2} \mid p, \theta_{1}, \mathbf{t}\right)$.

4) Repeat steps 2-3 N times. Now calculate Bayes estimator of $\phi$ under squared and LINEX loss functions, respectively, by

$$
\begin{gathered}
E(\phi \mid \mathbf{t})=(1 /(N-v)) \sum_{i=v+1}^{N} \phi\left(p^{i}, \theta_{1}^{i}, \theta_{2}^{i}\right), \\
E\left(\exp (-a \phi \mid \mathbf{t})=(1 /(N-v)) \sum_{i=v+1}^{N} \exp \left(-a \phi\left(p^{i}, \theta_{1}^{i}, \theta_{2}^{i}\right)\right),\right.
\end{gathered}
$$

where $v$ is the burn-in period.

\section{Numerical Computations}

A comparison between $M L$ and Bayes estimators, under either a squared error or a $L I N E X$ loss functions, is made using a Monte Carlo simulation study in the two following cases:

\subsection{Progressive Type-II Censored Order Statistics}

The progressive Type-II censored order statistics can be obtained from the GOS as a special case by taking $m_{i}=r_{i}$ for $i=1,2, \ldots, m-1$ and $k=r_{m}+1$. Therefore, the estimation results obtained in the above sections can be specialized to the progressive Type-II censored order statistics. Estimates of parameters, $R F$ and $H R F$ are computed and compared based on Monte Carlo simulation study according to the following steps:

1) For given values of $p, \theta_{1}$ and $\theta_{2}$, we generate progressively Type-II censored samples from the MTRD by using the algorithm described in Balakrishnan and Sandhu (1995), as follows:

- using $p, \theta_{1}$ and $\theta_{2}$, with different chooses of $n, m$ and $k$. We take in our consideration that the progressive censored order statistics $T_{1: m: n: k}^{R}, T_{2: m: n: k}^{R}, \ldots, T_{m: m: n: k}^{R}$ is a progressively Type-II censored sample from a population with $C D F(6)$.

- generate $m$ independent Uniform $(0,1)$ observations $w_{1}, w_{2}, \ldots, w_{m}$.

- determine the values of the censored scheme $r_{i}$, for $i=1,2, \ldots, m$.

- $\operatorname{set} E_{i}=1 /\left(i+\sum_{j=m-i+1}^{m} r_{j}\right)$ for $i=1,2, \ldots, m$.

- set $V_{i}=w_{i}^{E_{i}}$ for $i=1,2, \ldots, m$.

- set $U_{i, m, n} \equiv U_{i}=1-V_{m} . V_{m-1} \ldots V_{m-i+1}$ for $i=1,2, \ldots, m$. Then $U_{1}, U_{2}, \ldots, U_{m}$ is the progressively Type-II right censored sample from the Uniform $(0,1)$ distribution.

- for given values of parameters $\theta_{1}, \theta_{2}$ and mixing proportion $p$, set:

$$
U_{i}=p\left[1-e^{-\theta_{1} t_{i}^{2}}\right]+(1-p)\left[1-e^{-\theta_{2} t_{i}^{2}}\right] .
$$

For $i=1,2, \ldots, m$, the values of $t_{i}$ for each $U_{i}$ can be obtained numerically from (32). Then the resulting set $t_{1}, t_{2}, \ldots, t_{m}$ is the required progressively Type-II right censored sample from the MTRD.

Using the algorithm described above, a progressively Type-II censored samples of size $m$ with different censored schemes are randomly generated from sample of size $n$ simulated from the MTRD. 
2) The $M L$ estimates of the parameters $p, \theta_{1}$ and $\theta_{2}$ are obtained by solving the nonlinear equations (8), with $m_{i}=r_{i}, k=r_{m}+1, i=1,2, \ldots, m-1$, numerically.

3) Based on squared error and LINEX loss functions the Bayes estimates of the parameters, reliability and Hazard rate functions are computed, from (26) and (27), according to the above $M C M C$ method.

The above steps are repeated 500 times. The estimated risks $(E R)$ are computed by averaging the squared deviations over the 500 repetitions.

\subsection{Upper Record Values}

The upper record values can be obtained from the $G O S$ by taking $m_{i}=-1$ for $i=1,2, \ldots, n-1$ and $k=1$. In this case, $M L$ and Bayes estimates of parameters, $R F$ and $H R F$ are computed and compared based on Monte Carlo simulation study according to the following steps:
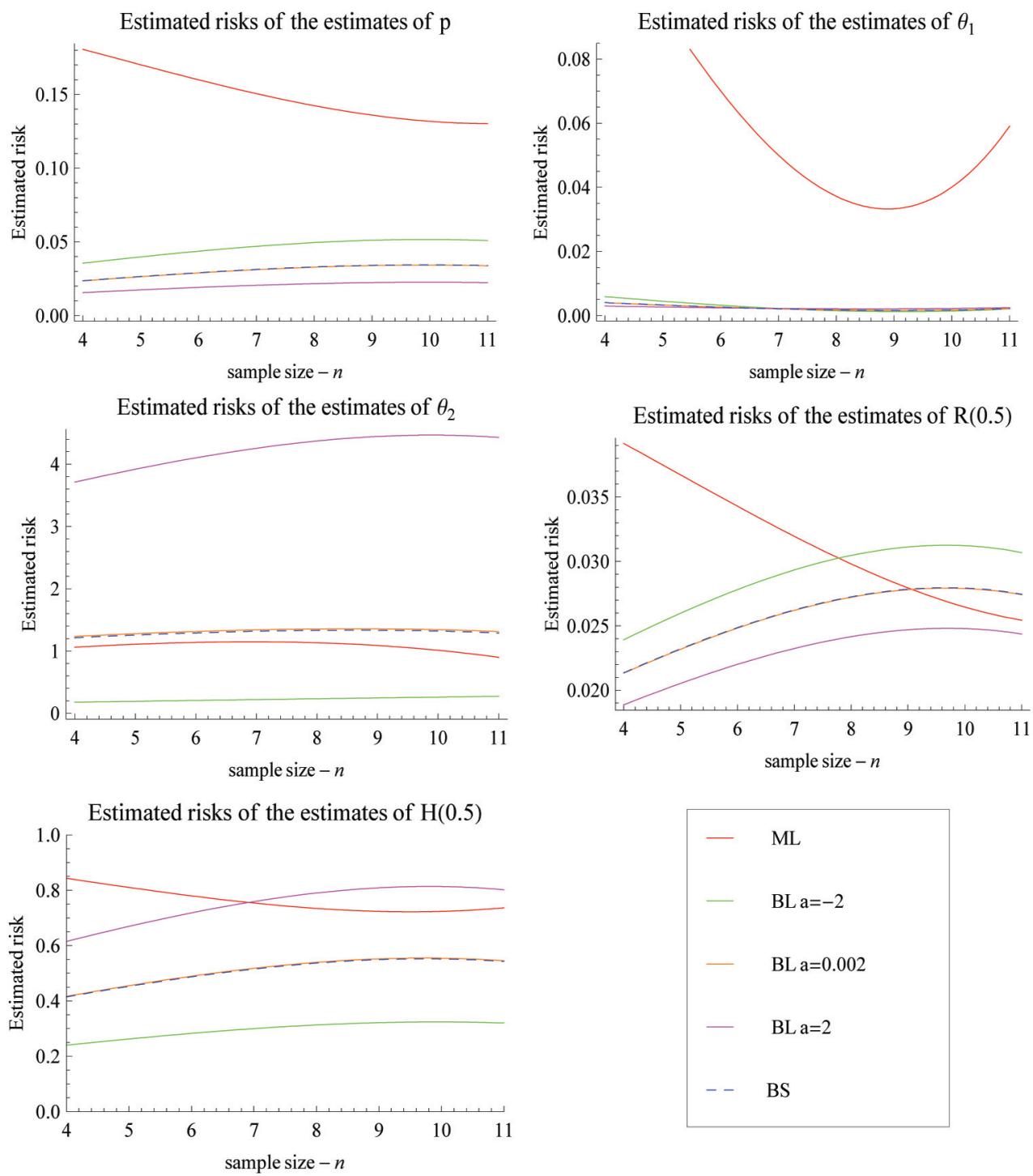

Figure 1. Estimated Risks $(E R)$ of the estimates of $\theta=\left(p, \theta_{1}, \theta_{2}\right), R(t)$ and $H(t)$ based on upper record values

1) For given values of $p, \theta_{1}$ and $\theta_{2}$, we generate $n=5,8,10$ upper record values from the MTRD.

2) The $M L$ estimates of the parameters $p, \theta_{1}$ and $\theta_{2}$ are obtained by solving the nonlinear equations (8), with $m_{i}=-1, k=1$, numerically.

3) Based on squared error and LINEX loss functions the Bayes estimates of the parameters, reliability and Hazard rate functions are computed, from (26) and (27), according to the above MCMC method. The above steps are repeated 1000 times. The estimated risks $(E R)$ are computed by averaging the squared deviations over the 1000 
repetitions.

The computational (our) results were computed by using Mathematica 6.0. Under conjugate prior the prior parameters chosen as $b_{1}=1.2, b_{2}=2.3, \alpha_{1}=2, \beta_{1}=0.3, \alpha_{2}=2, \beta_{2}=3$ which yield the generated values of $p=0.391789, \theta_{1}=0.307317$ and $\theta_{2}=3.33166$ (as the true values). The true values of $R(t)$ and $H(t)$ when $t=0.5$, are computed to be $R(0.5)=0.627253$ and $H(0.5)=1.58232$. While, under non-informative prior values of $\left(p, \theta_{1}, \theta_{2}\right)$ chosen as $(0.4,0.3,3)$. The true values of $(R(0.5), H(0.5))$ are computed to be $(0.654517,1.46916)$. The value of the shape parameter $a$ of the LINEX loss function is $a=(-2,0.02,2)$. The estimated risks $(E R)$ are displayed in Tables 1-2-3-4. Figures 1 and 2 represents the estimated risks of the estimates in the case of upper record values.
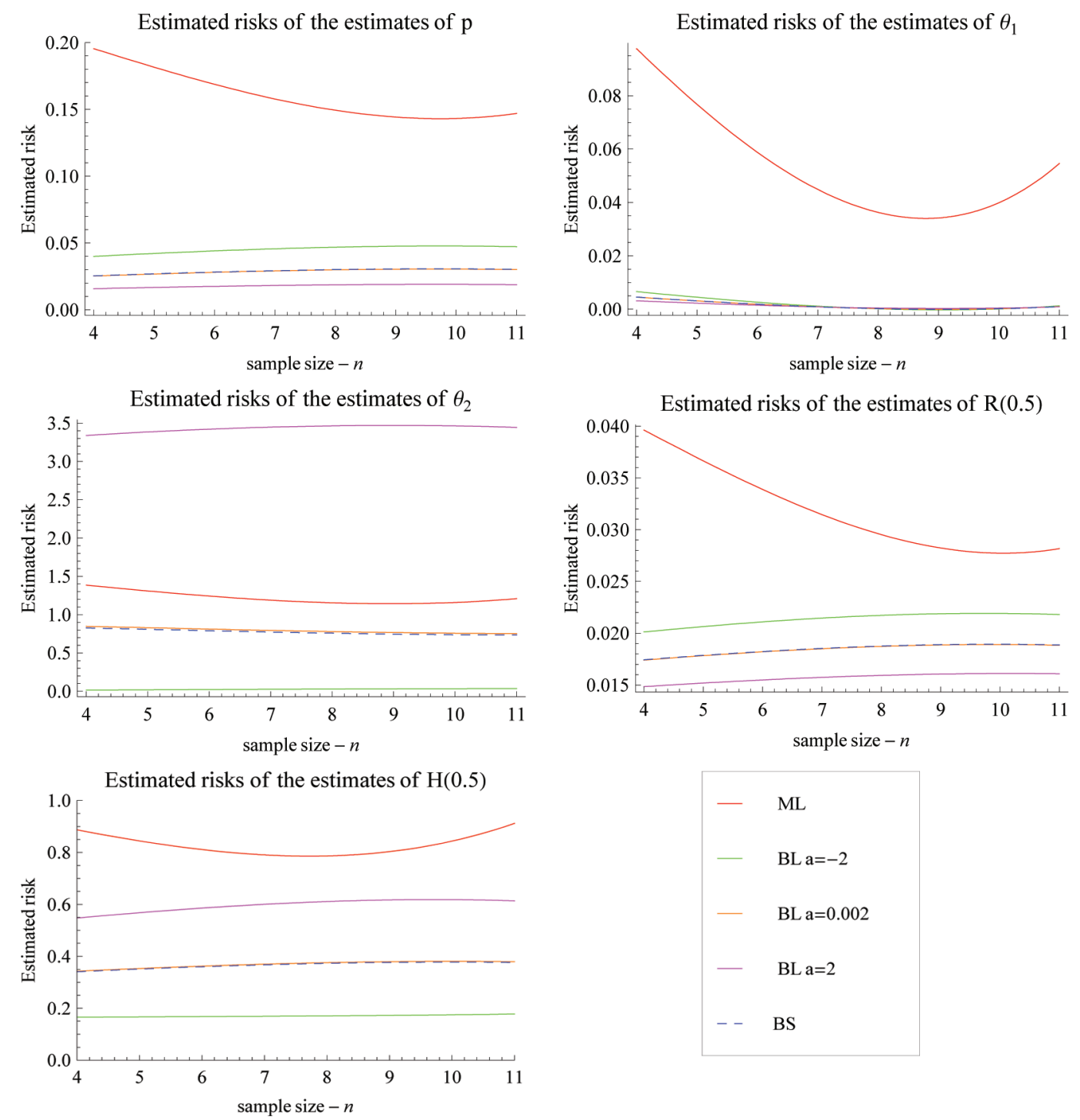

Figure 2. Estimated Risks $(E R)$ of the estimates of $\theta=\left(p, \theta_{1}, \theta_{2}\right), R(t)$ and $H(t)$ based on upper record values

\section{Concluding Remarks}

Based on GOS model, this paper proposes Bayesian and non-Bayesian approach to estimate the unknown parameters for the mixture of two-component Rayleigh model. Samples from heterogeneous populations that can be represented by a finite mixture of two-component Rayleigh model are subjected to Type II right progressively censoring and upper record values cases. The $M L$ and some Bayes methods are used to estimate the parameters, reliability and hazard rate functions. From the results of the simulation study we observe that: 
Table 1. (Progressively censored samples) $E R$ of the $M L$ estimates and the Bayes $(B S, B L)$ estimates assuming the conjugate prior of $p, \theta_{1}, \theta_{2}, R(0.5)$ and $H(0.5)$ for different $m, n$ and schemes

\begin{tabular}{|c|c|c|c|c|c|c|c|c|}
\hline \multirow[t]{2}{*}{$n$} & \multirow[t]{2}{*}{$m$} & \multirow[t]{2}{*}{ Scheme } & \multirow[t]{2}{*}{ Parameters } & \multirow[t]{2}{*}{$M L$} & \multirow[t]{2}{*}{$B S$} & \multicolumn{3}{|c|}{$B L$} \\
\hline & & & & & & $a=-2$ & $a=0.002$ & $a=2$ \\
\hline \multirow{15}{*}{50} & \multirow{15}{*}{25} & \multirow[t]{5}{*}{$\left(25,24^{* 0}\right)$} & $P$ & 0.003245 & 0.005342 & 0.003536 & 0.005365 & 0.008055 \\
\hline & & & $\theta_{1}$ & 0.67785 & 0.54289 & 0.8398 & 0.54036 & 0.3354 \\
\hline & & & $\theta_{2}$ & 0.43825 & 0.11166 & 0.12969 & 0.431641 & 0.65218 \\
\hline & & & $R(t=0.5)$ & 0.09767 & 0.05947 & 0.05757 & 0.05953 & 0.06114 \\
\hline & & & $H(t=0.5)$ & 0.839173 & 0.40463 & 0.524707 & 0.40454 & 0.50510 \\
\hline & & \multirow[t]{5}{*}{$\left(24^{* 0}, 25\right)$} & $P$ & 0.003137 & 0.005140 & 0.003397 & 0.005161 & 0.007764 \\
\hline & & & $\theta_{1}$ & 0.66734 & 0.54715 & 0.84843 & 0.54457 & 0.33613 \\
\hline & & & $\theta_{2}$ & 0.44965 & 0.05612 & 0.13305 & 0.43447 & 0.6539 \\
\hline & & & $R(t=0.5)$ & 0.09160 & 0.05778 & 0.056155 & 0.05784 & 0.05889 \\
\hline & & & $H(t=0.5)$ & 0.888689 & 0.402153 & 0.52249 & 0.40206 & 0.50192 \\
\hline & & \multirow[t]{5}{*}{$\left(12^{* 0}, 25,12^{* 0}\right)$} & $P$ & 0.003495 & 0.005146 & 0.003405 & 0.005167 & 0.007768 \\
\hline & & & $\theta_{1}$ & 0.71011 & 0.55162 & 0.085252 & 0.54905 & 0.34024 \\
\hline & & & $\theta_{2}$ & 0.443812 & 0.05585 & 0.12879 & 0.42776 & 0.64839 \\
\hline & & & $R(t=0.5)$ & 0.075615 & 0.04808 & 0.04649 & 0.048142 & 0.05914 \\
\hline & & & $H(t=0.5)$ & 0.86252 & 0.41210 & 0.533473 & 0.412899 & 0.502635 \\
\hline \multirow{15}{*}{50} & \multirow{15}{*}{35} & $\left(15,34^{* 0}\right)$ & $P$ & 0.000224 & 0.005202 & 0.003435 & 0.0052234 & 0.007861 \\
\hline & & & $\theta_{1}$ & 0.40911 & 0.47685 & 0.3435 & 0.5223 & 0.7861 \\
\hline & & & $\theta_{2}$ & 0.44669 & 0.041131 & 0.037051 & 0.043567 & 0.065858 \\
\hline & & & $R(t=0.5)$ & 0.04352 & 0.036785 & 0.03525 & 0.03685 & 0.04780 \\
\hline & & & $H(t=0.5)$ & 0.39355 & 0.106689 & 0.11706 & 0.106590 & 0.116211 \\
\hline & & $\left(34^{* 0}, 15\right)$ & $P$ & 0.018655 & 0.005111 & 0.0033806 & 0.0051322 & 0.007716 \\
\hline & & & $\theta_{1}$ & 0.7927 & 0.048203 & 0.077453 & 0.047956 & 0.382002 \\
\hline & & & $\theta_{2}$ & 0.415377 & 0.031153 & 0.013247 & 0.043822 & 0.066359 \\
\hline & & & $R(t=0.5)$ & 0.064502 & 0.03607 & 0.03477 & 0.03613 & 0.05674 \\
\hline & & & $H(t=0.5)$ & 0.938207 & 0.105817 & 0.116416 & 0.105716 & 0.104960 \\
\hline & & $\left(17^{* 0}, 15,17^{* 0}\right)$ & $P$ & 0.003343 & 0.0051459 & 0.0034011 & 0.0051673 & 0.00777 \\
\hline & & & $\theta_{1}$ & 0.61314 & 0.04841 & 0.07767 & 0.048163 & 0.38382 \\
\hline & & & $\theta_{2}$ & 0.43851 & 0.031173 & 0.011847 & 0.042113 & 0.06491 \\
\hline & & & $R(t=0.5)$ & 0.09116 & 0.036801 & 0.035594 & 0.036859 & 0.05752 \\
\hline & & & $H(t=0.5)$ & 0.46447 & 0.10739 & 0.11846 & 0.10729 & 0.10644 \\
\hline & & $\left(50,49^{* 0}\right)$ & $P$ & 0.000099 & 0.0048152 & 0.0029527 & 0.0049369 & 0.007266 \\
\hline & & & $\theta_{1}$ & 0.31932 & 0.050734 & 0.081869 & 0.050475 & 0.030445 \\
\hline & & & $\theta_{2}$ & 0.41668 & 0.010226 & 0.01062 & 0.038718 & 0.059884 \\
\hline & & & $R(t=0.5)$ & 0.007391 & 0.004301 & 0.004435 & 0.0047075 & 0.005429 \\
\hline & & & $H(t=0.5)$ & 0.302130 & 0.105334 & 0.10585 & 0.105236 & 0.105243 \\
\hline 100 & 50 & $\left(49^{* 0}, 50\right)$ & $P$ & 0.000214 & 0.005003 & 0.0032048 & 0.005125 & 0.007610 \\
\hline & & & $\theta_{1}$ & 0.385417 & 0.050729 & 0.082766 & 0.053472 & 0.032663 \\
\hline & & & $\theta_{2}$ & 0.42668 & 0.010134 & 0.0115348 & 0.038483 & 0.058634 \\
\hline & & & $R(t=0.5)$ & 0.007874 & 0.004659 & 0.004571 & 0.004606 & 0.005314 \\
\hline & & & $H(t=0.5)$ & 0.355113 & 0.105033 & 0.105358 & 0.105139 & 0.102095 \\
\hline & & $\left(20^{* 0}, 50,29^{* 0}\right)$ & $P$ & 0.000371 & 0.005176 & 0.003086 & 0.0055098 & 0.007265 \\
\hline & & & $\theta_{1}$ & 0.320971 & 0.053405 & 0.081019 & 0.052142 & 0.034789 \\
\hline & & & $\theta_{2}$ & 0.42669 & 0.010182 & 0.011466 & 0.040447 & 0.06167 \\
\hline & & & $R(t=0.5)$ & 0.006237 & 0.004372 & 0.004597 & 0.00468 & 0.005720 \\
\hline & & & $H(t=0.5)$ & 0.31214 & 0.10685 & 0.10756 & 0.10675 & 0.10678 \\
\hline & & $\left(20,79^{* 0}\right)$ & $P$ & 0.000014 & 0.004085 & 0.002361 & 0.0041057 & 0.006683 \\
\hline & & & $\theta_{1}$ & 0.277822 & 0.045999 & 0.075848 & 0.045744 & 0.024999 \\
\hline & & & $\theta_{2}$ & 0.346678 & 0.001115 & 0.003173 & 0.033384 & 0.055403 \\
\hline & & & $R(t=0.5)$ & 0.006750 & 0.003761 & 0.003604 & 0.003767 & 0.005002 \\
\hline & & & $H(t=0.5)$ & 0.26320 & 0.104754 & 0.115234 & 0.090465 & 0.094745 \\
\hline 100 & 80 & $\left(79^{* 0}, 20\right)$ & $P$ & 0.000149 & 0.004237 & 0.002465 & 0.004259 & 0.006902 \\
\hline & & & $\theta_{1}$ & 0.28209 & 0.04592 & 0.075811 & 0.045668 & 0.02492 \\
\hline & & & $\theta_{2}$ & 0.346678 & 0.001565 & 0.002703 & 0.032736 & 0.054899 \\
\hline & & & $R(t=0.5)$ & 0.006451 & 0.003902 & 0.003727 & 0.003908 & 0.005041 \\
\hline & & & $H(t=0.5)$ & 0.241003 & 0.104896 & 0.125183 & 0.094801 & 0.095045 \\
\hline & & $\left(40^{* 0}, 20,39^{* 0}\right)$ & $P$ & 0.000149 & 0.004085 & 0.002361 & 0.004106 & 0.006683 \\
\hline & & & $\theta_{1}$ & 0.277822 & 0.045999 & 0.075847 & 0.045744 & 0.024999 \\
\hline & & & $\theta_{2}$ & 0.346678 & 0.0011146 & 0.003172 & 0.033384 & 0.055403 \\
\hline & & & $R(t=0.5)$ & 0.006750 & 0.003761 & 0.003604 & 0.003767 & 0.004866 \\
\hline & & & $H(t=0.5)$ & 0.255123 & 0.112615 & 0.112844 & 0.092519 & 0.092512 \\
\hline
\end{tabular}

$B L \equiv$ Bayes (LINEX loss function); $B S \equiv$ Bayes (squared error loss function). 
Table 2. (Progressively censored samples) $E R$ of the $M L$ estimates and the Bayes $(B S, B L)$ estimates assuming the noninformative prior of $p, \theta_{1}, \theta_{2}, R(0.5)$ and $H(0.5)$ for different $m, n$ and schemes

\begin{tabular}{|c|c|c|c|c|c|c|c|c|}
\hline \multirow[t]{2}{*}{$n$} & \multirow[t]{2}{*}{$m$} & \multirow[t]{2}{*}{ Scheme } & \multirow[t]{2}{*}{ Parameters } & \multirow[t]{2}{*}{$M L$} & \multirow[t]{2}{*}{$B S$} & \multicolumn{3}{|c|}{$B L$} \\
\hline & & & & & & $a=-2$ & $a=0.002$ & $a=2$ \\
\hline \multirow{15}{*}{50} & \multirow{15}{*}{25} & \multirow[t]{5}{*}{$\left(25,24^{* 0}\right)$} & $P$ & 0.000275 & 0.004360 & 0.00633 & 0.009191 & 0.006308 \\
\hline & & & $\theta_{1}$ & 0.91462 & 0.08854 & 0.05759 & 0.03607 & 0.05785 \\
\hline & & & $\theta_{2}$ & 1 & 0.045487 & 0.014611 & 0.005531 & 0.011142 \\
\hline & & & $R(t=0.5)$ & 0.018702 & 0.009628 & 0.00990 & 0.011494 & 0.00989 \\
\hline & & & $H(t=0.5)$ & 0.867288 & 0.21134 & 0.184647 & 0.184727 & 0.184773 \\
\hline & & \multirow[t]{5}{*}{$\left(24^{* 0}, 25\right)$} & $P$ & 0.000269 & 0.004403 & 0.006372 & 0.0092145 & 0.006348 \\
\hline & & & $\theta_{1}$ & 0.975792 & 0.090199 & 0.05867 & 0.036548 & 0.058939 \\
\hline & & & $\theta_{2}$ & 1 & 0.048138 & 0.015518 & 0.005860 & 0.00554 \\
\hline & & & $R(t=0.5)$ & 0.019622 & 0.009806 & 0.010031 & 0.011569 & 0.010023 \\
\hline & & & $H(t=0.5)$ & 0.925593 & 0.215921 & 0.18774 & 0.186478 & 0.187879 \\
\hline & & \multirow{5}{*}{$\left(12^{* 0}, 25,12^{* 0}\right)$} & $P$ & 0.000515 & 0.004465 & 0.006469 & 0.009359 & 0.006444 \\
\hline & & & $\theta_{1}$ & 0.93515 & 0.08980 & 0.058226 & 0.036283 & 0.058495 \\
\hline & & & $\theta_{2}$ & 1 & 0.047101 & 0.015102 & 0.005692 & 0.005514 \\
\hline & & & $R(t=0.5)$ & 0.018367 & 0.009844 & 0.010099 & 0.011683 & 0.010090 \\
\hline & & & $H(t=0.5)$ & 0.853737 & 0.216007 & 0.188208 & 0.187661 & 0.188343 \\
\hline \multirow{15}{*}{50} & \multirow{15}{*}{35} & $\left(15,34^{* 0}\right)$ & $P$ & 0.000276 & 0.004458 & 0.006457 & 0.009343 & 0.006433 \\
\hline & & & $\theta_{1}$ & 0.922268 & 0.091183 & 0.059727 & 0.037789 & 0.059996 \\
\hline & & & $\theta_{2}$ & 1 & 0.047677 & 0.015509 & 0.005901 & 0.005562 \\
\hline & & & $R(t=0.5)$ & 0.018958 & 0.009883 & 0.010152 & 0.011745 & 0.010143 \\
\hline & & & $H(t=0.5)$ & 0.882936 & 0.21764 & 0.190233 & 0.189985 & 0.19037 \\
\hline & & $\left(34^{* 0}, 15\right)$ & $P$ & 0.000474 & 0.004481 & 0.006509 & 0.009444 & 0.006485 \\
\hline & & & $\theta_{1}$ & 0.93531 & 0.090395 & 0.059027 & 0.037214 & 0.059295 \\
\hline & & & $\theta_{2}$ & 1 & 0.046569 & 0.015072 & 0.005726 & 0.005638 \\
\hline & & & $R(t=0.5)$ & 0.01830 & 0.009859 & 0.0101645 & 0.011806 & 0.010155 \\
\hline & & & $H(t=0.5)$ & 0.84993 & 0.216491 & 0.189719 & 0.190196 & 0.189845 \\
\hline & & $\left(17^{* 0}, 15,17^{* 0}\right)$ & $P$ & 0.000401 & 0.004443 & 0.006431 & 0.009301 & 0.006407 \\
\hline & & & $\theta_{1}$ & 0.92685 & 0.090563 & 0.058979 & 0.036915 & 0.059251 \\
\hline & & & $\theta_{2}$ & 1 & 0.048117 & 0.015634 & 0.005935 & 0.005563 \\
\hline & & & $R(t=0.5)$ & 0.018387 & 0.009863 & 0.01011 & 0.011674 & 0.010101 \\
\hline & & & $H(t=0.5)$ & 0.85276 & 0.217056 & 0.189157 & 0.188294 & 0.189295 \\
\hline & & $\left(50,49^{* 0}\right)$ & $P$ & 0.000227 & 0.004266 & 0.006066 & 0.008953 & 0.006142 \\
\hline & & & $\theta_{1}$ & 0.90859 & 0.089084 & 0.057725 & 0.034868 & 0.057993 \\
\hline & & & $\theta_{2}$ & 1 & 0.044692 & 0.013094 & 0.005427 & 0.00523 \\
\hline & & & $R(t=0.5)$ & 0.017199 & 0.0096387 & 0.009712 & 0.010704 & 0.010103 \\
\hline & & & $H(t=0.5)$ & 0.80632 & 0.210004 & 0.18875 & 0.181498 & 0.18888 \\
\hline 100 & 50 & $\left(49^{* 0}, 50\right)$ & $P$ & 0.000155 & 0.004133 & 0.006185 & 0.009111 & 0.006061 \\
\hline & & & $\theta_{1}$ & 0.91742 & 0.08977 & 0.057582 & 0.035839 & 0.057848 \\
\hline & & & $\theta_{2}$ & 1 & 0.044688 & 0.013437 & 0.005256 & 0.005364 \\
\hline & & & $R(t=0.5)$ & 0.017731 & 0.009612 & 0.009754 & 0.011512 & 0.00995 \\
\hline & & & $H(t=0.5)$ & 0.80107 & 0.21064 & 0.18720 & 0.18678 & 0.18733 \\
\hline & & $\left(20^{* 0}, 50,29^{* 0}\right)$ & $P$ & 0.000224 & 0.004239 & 0.006158 & 0.009153 & 0.006234 \\
\hline & & & $\theta_{1}$ & 0.88808 & 0.089878 & 0.057612 & 0.035851 & 0.057879 \\
\hline & & & $\theta_{2}$ & 1 & 0.044694 & 0.013433 & 0.00535 & 0.005299 \\
\hline & & & $R(t=0.5)$ & 0.016517 & 0.009669 & 0.010438 & 0.011751 & 0.010134 \\
\hline & & & $H(t=0.5)$ & 0.801348 & 0.210222 & 0.185989 & 0.187079 & 0.188118 \\
\hline & & $\left(20,79^{* 0}\right)$ & $P$ & 0.000187 & 0.0043384 & 0.006149 & 0.0091904 & 0.006126 \\
\hline & & & $\theta_{1}$ & 0.90281 & 0.088638 & 0.059406 & 0.035764 & 0.054673 \\
\hline & & & $\theta_{2}$ & 1 & 0.045035 & 0.013406 & 0.037764 & 0.005473 \\
\hline & & & $R(t=0.5)$ & 0.0176969 & 0.009685 & 0.010029 & 0.0115935 & 0.0100201 \\
\hline & & & $H(t=0.5)$ & 0.804349 & 0.210819 & 0.188241 & 0.1879 & 0.188375 \\
\hline 100 & 80 & $\left(79^{* 0}, 20\right)$ & $P$ & 0.000273 & 0.004381 & 0.006298 & 0.009207 & 0.006270 \\
\hline & & & $\theta_{1}$ & 0.912554 & 0.088357 & 0.059200 & 0.035603 & 0.005566 \\
\hline & & & $\theta_{2}$ & 1 & 0.046335 & 0.013266 & 0.005369 & 0.011089 \\
\hline & & & $R(t=0.5)$ & 0.017935 & 0.009684 & 0.010169 & 0.011788 & 0.010159 \\
\hline & & & $H(t=0.5)$ & 0.80684 & 0.210059 & 0.190006 & 0.190344 & 0.190134 \\
\hline & & $\left(40^{* 0}, 20,39^{* 0}\right)$ & $P$ & 0.000257 & 0.004345 & 0.006243 & 0.009233 & 0.006219 \\
\hline & & & $\theta_{1}$ & 0.894361 & 0.088981 & 0.059850 & 0.036156 & 0.055114 \\
\hline & & & $\theta_{2}$ & 1 & 0.045609 & 0.013513 & 0.005398 & 0.00138 \\
\hline & & & $R(t=0.5)$ & 0.017017 & 0.009658 & 0.010140 & 0.011747 & 0.010131 \\
\hline & & & $H(t=0.5)$ & 0.80785 & 0.210085 & 0.190130 & 0.190330 & 0.190262 \\
\hline
\end{tabular}

$B L \equiv$ Bayes (LINEX loss function); BS $\equiv$ Bayes (squared error loss function).

1) All of the results obtained in this article in case of Type II right progressively censoring can be specialized to 
both the complete sample case by taking $\left(m=n, r_{i}=0, i=1,2,3,4, \ldots m\right)$, and the Type-II right censored sample for $\left(r_{i}=0, i=1,2,3,4, \ldots m-1, r_{m}=n-m\right)$.

2) From Table 1, we see that the Bayes estimators relative to asymmetric loss functions (LINEX), are sensitive to the value of the shape parameters $a$, for small $a$ the Bayes estimates under the LINEX loss function $(B L)$, are very close to the Bayes estimates under the squared error loss function. The Bayes estimates of $R F$ under the $L I N E X$ loss function $(B L)$, when $a=-2$ have the smallest $E R^{\prime} s$ as compared with their corresponding estimates. While, the Bayes estimates of $\theta_{1}, \theta_{2}$ and $H R F$ have the smallest $E R^{\prime} s$ as compared with their corresponding $M L$ estimates. In most of the considered cases, it is observed that the $M L$ estimates of $p$ have the smallest $E R^{\prime} s$ as compared with their corresponding Bayes estimates.

3) Also from Table 1, as the proportion $m / n$ increases, the $E R^{\prime} s$ reduce significantly. We observed that for large sample size, the symmetric and asymmetric Bayes estimates are better than the $M L$ estimates.

4) Table 2 shows that the Bayes estimates of $\theta_{1}, \theta_{2}$ and $H R F$ under the LINEX loss function have the smallest $E R^{\prime} s$ as compared with their corresponding estimates. While, the Bayes estimates of $R F$ under the squared error loss function have the smallest $E R^{\prime} s$ as compared with their corresponding estimates. In most of the considered cases, Table 2 shows that the $M L$ estimates of $p$ have the smallest $E R^{\prime} s$ as compared with their corresponding Bayes estimates.

Table 3. (Upper record values) $E R$ of the $M L$ estimates and the Bayes ( $B S, B L$ ) estimates assuming the conjugate prior of $p, \theta_{1}, \theta_{2}, R(0.5)$ and $H(0.5)$ for different sample size

\begin{tabular}{ccccccc}
\hline$n$ & Parameters & $M L$ & $B S$ & & $B L$ & \\
& & & & $a=-2$ & $a=0.002$ & $a=2$ \\
\hline 5 & $P$ & 0.170122 & 0.0264868 & 0.0397904 & 0.0263757 & 0.0174207 \\
& $\theta_{1}$ & 0.0956744 & 0.00328384 & 0.00447608 & 0.00327487 & 0.00266551 \\
& $\theta_{2}$ & 1.109 & 1.2566 & 0.19321 & 1.27885 & 3.91814 \\
& $R(t=0.5)$ & 0.0367232 & 0.0232053 & 0.0259773 & 0.023178 & 0.020526 \\
& $H(t=0.5)$ & 0.810279 & 0.45288 & 0.262485 & 0.455084 & 0.669544 \\
\hline 8 & $P$ & 0.142407 & 0.0329758 & 0.0494921 & 0.0328373 & 0.0216422 \\
& $\theta_{1}$ & 0.0371982 & 0.00174507 & 0.00150609 & 0.00174812 & 0.00212429 \\
& $\theta_{2}$ & 1.13347 & 1.33192 & 0.234972 & 1.35535 & 4.37182 \\
& $R(t=0.5)$ & 0.0298045 & 0.027248 & 0.0304673 & 0.0272163 & 0.0241562 \\
& $H(t=0.5)$ & 0.734561 & 0.536832 & 0.313168 & 0.539407 & 0.790005 \\
\hline 10 & $P$ & 0.131772 & 0.0344254 & 0.0516252 & 0.0342812 & 0.0226098 \\
& $\theta_{1}$ & 0.0400329 & 0.00174828 & 0.00139018 & 0.00175243 & 0.00223124 \\
& $\theta_{2}$ & 1.01259 & 1.32074 & 0.261058 & 1.3437 & 4.46605 \\
& $R(t=0.5)$ & 0.0264525 & 0.0279305 & 0.0312235 & 0.0278982 & 0.0247848 \\
& $H(t=0.5)$ & 0.723828 & 0.551947 & 0.32409 & 0.554589 & 0.813456 \\
\hline
\end{tabular}

$B L \equiv$ Bayes (LINEX loss function); $B S \equiv$ Bayes (squared error loss function).

Table 4. (Upper record values) $E R$ of the $M L$ estimates and the Bayes $(B S, B L)$ estimates assuming the noninformative prior of $p, \theta_{1}, \theta_{2}, R(0.5)$ and $H(0.5)$ for different sample size

\begin{tabular}{ccccccc}
\hline$n$ & Parameters & $M L$ & $B S$ & & $B L$ \\
& & & & $a=-2$ & $a=0.002$ & $a=2$ \\
\hline 5 & $P$ & 0.181571 & 0.0269282 & 0.0421724 & 0.0268018 & 0.0167192 \\
& $\theta_{1}$ & 0.0768179 & 0.00311924 & 0.00447972 & 0.0031081 & 0.0022672 \\
& $\theta_{2}$ & 1.30989 & 0.808531 & 0.0191031 & 0.830045 & 3.385 \\
& $R(t=0.5)$ & 0.036651 & 0.0178594 & 0.0206431 & 0.0178319 & 0.0151835 \\
& $H(t=0.5)$ & 0.844474 & 0.350973 & 0.166689 & 0.35319 & 0.567843 \\
\hline 8 & $P$ & 0.149364 & 0.0300759 & 0.0468695 & 0.0299361 & 0.0187382 \\
& $\theta_{1}$ & 0.0362808 & 0.000218835 & 0.000147729 & 0.000220181 & 0.00043849 \\
& $\theta_{2}$ & 1.15463 & 0.758679 & 0.0296448 & 0.778903 & 3.46421 \\
& $R(t=0.5)$ & 0.0295189 & 0.0187535 & 0.021728 & 0.0187244 & 0.0159262 \\
& $H(t=0.5)$ & 0.786627 & 0.373189 & 0.170778 & 0.375601 & 0.610974 \\
\hline 10 & $P$ & 0.143208 & 0.0306175 & 0.0477189 & 0.0304752 & 0.0190788 \\
& $\theta_{1}$ & 0.0399092 & 0.000177435 & 0.0000962643 & 0.000178922 & 0.00041641 \\
& $\theta_{2}$ & 1.15942 & 0.738898 & 0.0342423 & 0.758505 & 3.4634 \\
& $R(t=0.5)$ & 0.0277292 & 0.0189317 & 0.0219134 & 0.0189025 & 0.0161039 \\
& $H(t=0.5)$ & 0.84348 & 0.378136 & 0.174893 & 0.380563 & 0.618301 \\
\hline
\end{tabular}

$B L \equiv$ Bayes (LINEX loss function); BS $\equiv$ Bayes (squared error loss function). 
5) From Tables 3 and 4, we see that in most of the considered cases, the Bayes estimates of $p, \theta_{1}, R F$ and $H R F$ have the smallest $E R^{\prime} s$ as compared with their corresponding $M L$ estimates. While, the Bayes estimates of $\theta_{2}$ under the LINEX loss function $(B L)$ when $a=-2$ have the smallest $E R^{\prime} s$ as compared with their corresponding estimates. Also, it is observed that the Bayes estimates of $p$ and $R F$ under the LINEX loss function perform best when the value of the shape parameters $a$ is large. While, the Bayes estimates of $\theta_{1}, \theta_{2}$ and $H R F$ under the LINEX loss function perform best when the value of the shape parameters $a$ is small.

\section{References}

Aboeleneen, Z. A. (2010). Inference for Weibull distribution under generalized order statistics. Mathematics and Computers in Simulation, 81, 26-36. http://dx.doi.org/10.1016/j.matcom.2010.06.013

Abu El Fotouh, S. (2011). Estimation for the parameters of the Weibull Extension model based on generalized order statistics. Int. J. Contemp. Math. Sciences, 6(36), 1749-1760.

Ahmad, A. A. (2007). Relations for single and product moments of generalized order statistics from doubly truncated Burr type XII distribution. J. Egypt. Math. Soc., 15(1), 117-128.

Ahmad, A. A. (2008). Single and product moments of generalized order statistics from linear exponential distribution. Commun. Statist. Theory. Meth., 37(8), 1162- 1172. http://dx.doi.org/10.1080/03610920701713344

Ahmad, K. E. (1988). Identifiability of finite mixtures using a new transform. Ann. Inst. Statist. Math., 40(2), 261-265. http://dx.doi.org/10.1007/BF00052342

Ahmad, K. E., Moustafa, H. M., \& Abd-El-Rahman, A. M. (1997). Approximate Bayes estimation for mixtures of two Weibull distributions under type II censoring. Journal of Statistical Computation and Simulation, 58(3), 269-285. http://dx.doi.org/10.1080/00949659708811835

Ahsanullah, M. (1996). Generalized order statistics from two parameter uniform distribution. Commun. Statist. Theory. Meth., 25(10), 2311-2318. http://dx.doi.org/10.1080/03610929608831840

Ahsanullah, M. (2000). Generalized order statistics from exponential distributiuon. J. Statist. Plann. Infer., 85, 85-91. http://dx.doi.org/10.1016/S0378-3758(99)00068-3

AL-Hussaini, E. K. (2004). Generalized order statistics: Prospective and Appli- cations. J. of Appl. Statist. Sci., $13(1), 59-85$.

AL-Hussaini, E. K., \& Ahmad, A. A. (2003). On Bayesian predictive distributions of generalized order statistics. Metrika, 57, 165-176. http://dx.doi.org/10.1007/s001840200207

AL-Hussaini, E. K., \& Ahmad, K. E. (1981). On the identifiability of finite mixtures of distributions. IEEE Trans. Inform. Theory, IT-27(5), 664- 668.

Ateya, S. F., \& Ahmad, A. A. (2011). Inferences based on generalized order statistics under truncated Type I generalized logistic distribution. Statistics, 45(4), 389-402. http://dx.doi.org/10.1080/02331881003650149

Attia, A. F. (1993). On estimation for mixtures of 2 Rayleigh distribution with censoring. Microelectronics and Reliability, 33(6), 859-867. http://dx.doi.org/10.1016/0026-2714(93)90259-2

Balakrishnan, N., \& Sandhu, R. A. (1995). A simple simulational algorithm for generating progressive Type-II censored samples. Amer. Statistician, 49, 229-230.

Cramer, E., \& Kamps, U. (2000). Relations for expectations of functions of generalized order statistics. J. Statist. Plann. and Infer., 89(1-2), 79-89. http://dx.doi.org/10.1016/S0378-3758(00)00074-4

Everitt, B. S., \& Hand, D. J. (1981). Finite Mixture Distributions. University Press, Cambridge. http://dx.doi.org/10.1007/978-94-009-5897-5

Habibullah, M., \& Ahsanullah, M. (2000). Estimation of parameters of a Pareto distribution by generalized order statistics. Commun. Statist. Theor. Meth., 29(7), 1597-1609.

Jaheen, Z. F. (2002). On Bayesian prediction of generalized order statistics. J. Statist. Theor. Appl., 1(3), $191-204$.

Jaheen, Z. F. (2005a). Estimation based on generalized order statistics from the Burr model. Commun. Statist.Theor. Meth., 34, 785-794. http://dx.doi.org/10.1081/STA-200054408

Jaheen, Z. F. (2005b). On record statistics from a mixture of two exponential distributions. J. Statist. Comput. 
Simul., 75, 1-11. http://dx.doi.org/10.1080/00949650410001646924

Jaheen, Z. F., \& Al Harbi, M. M. (2010). Bayesian estimation for the exponentiated Weibull model via Markov chain Monte Carlo simulation. Communications in Statistics-Simulation and Computation (Accepted and to appear).

Kamps, U. (1995). A Concept of generalized order statistics. J. Statist Plann Inference, 48, 1-23. http://dx.doi.org/10.1016/0378-3758(94)00147-N

Kamps, U., \& Gather, U. (1997). Characteristic property of generalized order statistics for exponential distributions. Appl. Math. (Warsaw), 24(4), 383-391.

McLachlan, G. J., \& Basford, K. E. (1988). Mixture Models: Inferences and Applications to Clustering. Marcel Dekker, New York.

Papadapoulos, A. S., \& Padgett, W. J. (1986). On Bayes estimation for mixtures of two exponential-life-distributions from right-censored samples. IEEE Trans. http://dx.doi.org/10.1109/TR.1986.4335364

Polovko, A. M. (1968). Fundamentals of Reliability Theory. New York: Academic Press.

Press, S. J. (2003). Subjective and Objective Bayesian Statistics: Principles, Models and Applications. Wiley.

Ren, Z., Wang, G. Y., Chen, Q. B., \& Li, H. B. (2011). Modeling and Simulation of Rayleigh fading, path loss, and shadowing fading for wireless mobile networks. Simulation Modeling Practice and Theory, 19, 626-637. http://dx.doi.org/10.1016/j.simpat.2010.10.001

Saleem, M., \& Aslam, M. (2008a). Bayesian analysis of the two component mixture of the Rayleigh distribution assuming the uniform and the Jeffreys priors. J. App. Statist. Scien., 16(4), 105-113.

Saleem, M., \& Aslam, M. (2008b). On prior selection for the mixture of Rayleigh distribution using predictive Intervals. Pak. J. Statist., 24(1), 21-35.

Saleem, M., \& Irfan, M. (2010). On properties of the Bayes estimates of the Rayleigh mixture parameters: a simulation study. Pak. J. Statist., 26(3), 547-555.

Soliman, A. A. (2006). Estimators for the finite mixture of Rayleigh model based on progressively censored data. Communications in Statistics-Theory and Methods, http://dx.doi.org/10.1080/03610920500501379

Takeshi Yamane. (1998). Application of the Rayleigh distribution to size selectivity of small prawn pots for the oriental river prawn, Macrobrachium nipponense. Fisheries Research, 36, 27-33. http://dx.doi.org/10.1016/S0165-7836(98)00084-8

Teicher, H. (1963). Identifiability of finite mixtures. Ann. Math. Statist., 34, 1265-1269. http://dx.doi.org/10.1214/aoms/1177703862

Titterington, D. M., Smith, A. F. M., \& Makov, U. E. (1985). Statistical Analysis of Finite Mixture Distributions. New York: John Wiley and Sons.

Upadhyay, S. K., \& Gupta, A. (2010). A Bayes analysis of modified Weibull distribution via Markov chain Monte Carlo simulation. Journal of Statistical Computation and Simulation, 80(3), 241-254. http://dx.doi.org/10.1080/00949650802600730

Upadhyay, S. K., Vasishta, N., \& Smith, A. F. M. (2001). Bayes inference in life testing and reliability via Markov chain Monte Carlo simulation. Sankhya A, 63(1), 15-40. 